\title{
Lazy Cops and Robbers played on random graphs and graphs on surfaces
}

\author{
Deepak Bal, Anthony Bonato*, \\ William B. Kinnersley, and Pawee Praeat*
}

\begin{abstract}
We consider a variant of the game of Cops and Robbers, called Lazy Cops and Robbers, where at most one cop can move in any round. The lazy cop number is the analogue of the usual cop number for this game. Lazy Cops and Robbers was recently introduced by Offner and Ojakian, who provided asymptotic upper and lower bounds on the analogue of the cop number of the hypercube. By investigating expansion properties, we provide asymptotically almost sure bounds on the lazy cop number of binomial random graphs $\mathcal{G}(n, p)$ for a wide range of $p=p(n)$. We provide an upper bound for the lazy cop number of graphs with genus $g$ by using the Gilbert-Hutchinson-Tarjan separator theorem.
\end{abstract}

AMS 2000 SUBJECT CLASSIFICATIONS: 05C57, 05C80.

KEYWORDS AND PHRASES: Cops and Robbers, vertex-pursuit games, random graphs, domination, adjacency property, graph genus.

\section{Introduction}

The game of Cops and Robbers (defined, along with all the standard notation, at the end of this section) is usually studied in the context of the cop number, the minimum number of cops needed to ensure a winning strategy. The cop number is often challenging to analyze; establishing upper bounds for this parameter is the focus of Meyniel's conjecture that the cop number of a connected $n$-vertex graph is $O(\sqrt{n})$. For additional background on Cops and Robbers and Meyniel's conjecture, see the book [11] and the surveys $[3,6,7]$.

A number of variants of Cops and Robbers have been studied. For example, we may allow a cop to capture the robber from a distance $k$, where $k$ is a non-negative integer [8,9], play on edges [13], allow one or both players to move with different speeds $[2,14]$ or to teleport, allow the robber to capture the cops [10], or make the robber invisible or drunk [17, 18]. See Chapter 8 of [11] for a non-comprehensive survey of variants of Cops and Robbers.

\footnotetext{
${ }^{*}$ Supported by grants from NSERC and Ryerson.
} 
We are interested in slowing the cops down to create a situation akin to chess, where at most one chess piece can move in a round. Hence, our focus in the present article is a recent variant introduced by Offner and Ojakian [21], where at most one cop can move in any given round. We refer to this variant, whose formal definition appears in Section 1.1, as Lazy Cops and Robbers; the analogue of the cop number is called the lazy cop number, and is written $c_{L}(G)$ for a graph $G$. In [21] it was proved for the hypercube $Q_{n}$ that $2^{\lfloor\sqrt{n} / 20\rfloor} \leq c_{L}\left(Q_{n}\right)=O\left(2^{n} \log n / n^{3 / 2}\right)$. The lower bound was recently improved by the authors of this paper [4]. Now, it is known that $c_{L}\left(Q_{n}\right)=\Omega\left(2^{n} / n^{5 / 2+\varepsilon}\right)$ for any $\varepsilon>0$. We mention in passing that [21] introduced a number of variants of Cops and Robbers, in which some fixed number of cops (perhaps more than one) can move in a given round. We focus here on the extreme case in which only one cop moves in each round, but it seems likely that our techniques generalize to other variants.

We present two results on Lazy Cops and Robbers and the lazy cop number. In Theorem 2.1 we provide asymptotically almost sure bounds on the lazy cop number for binomial random graphs $\mathcal{G}(n, p)$ for a wide range of $p=p(n)$. We do this by examining typical expansion properties of such graphs. In Theorem 3.2, we provide an upper bound for graphs of genus $g$ using the Gilbert-Hutchinson-Tarjan separator theorem [15].

\subsection{Definitions and notation}

We consider only finite, undirected graphs in this paper. For background on graph theory, the reader is directed to [27].

The game of Cops and Robbers was independently introduced in [20, 25] and the cop number was introduced in [1]. The game is played on a reflexive graph; that is, each vertex has at least one loop. Multiple edges are allowed, but make no difference to the play of the game, so we always assume there is exactly one edge joining adjacent vertices. There are two players, consisting of a set of cops and a single robber. The game is played over a sequence of discrete time-steps or turns, with the cops going first on turn 0 and then playing on alternate time-steps. A round of the game is a cop move together with the subsequent robber move. The cops and robber occupy vertices; for simplicity, we often identify the player with the vertex they occupy. We refer to the set of cops as $C$ and the robber as $R$. When a player is ready to move in a round they must move to a neighbouring vertex. Because of the loops, players can pass, or remain on their own vertices. Observe that any subset of $C$ may move in a given round. The cops win if after some finite number of rounds, one of them can occupy the same vertex as the robber (in a reflexive 
graph, this is equivalent to the cop landing on the robber). This is called a capture. The robber wins if he can evade capture indefinitely. A winning strategy for the cops is a set of rules that if followed, result in a win for the cops. A winning strategy for the robber is defined analogously. As stated earlier, the game of Lazy Cops and Robbers is defined almost exactly as Cops and Robbers, with the exception that exactly one cop moves in any round.

If we place a cop at each vertex, then the cops are guaranteed to win. Therefore, the minimum number of cops required to win in a graph $G$ is a well-defined positive integer, named the lazy cop number of the graph $G$. We write $c_{L}(G)$ for the lazy cop number of a graph $G$.

\section{Random graphs}

In this section, we consider the game played on binomial random graphs. The random graph $\mathcal{G}(n, p)$ consists of the probability space $(\Omega, \mathcal{F}, \mathbb{P})$, where $\Omega$ is the set of all graphs with vertex set $\{1,2, \ldots, n\}, \mathcal{F}$ is the family of all subsets of $\Omega$, and for every $G \in \Omega$,

$$
\mathbb{P}(G)=p^{|E(G)|}(1-p)^{\left(\begin{array}{c}
n \\
2
\end{array}\right)-|E(G)|} .
$$

This space may be viewed as the set of outcomes of $\left(\begin{array}{l}n \\ 2\end{array}\right)$ independent coin flips, one for each pair $(u, v)$ of vertices, where the probability of success (that is, adding edge $u v$ ) is $p$. Note that $p=p(n)$ may (and usually does) tend to zero as $n$ tends to infinity. All asymptotics throughout are as $n \rightarrow$ $\infty$ (we emphasize that the notations $o(\cdot)$ and $O(\cdot)$ refer to functions of $n$, not necessarily positive, whose growth is bounded). We say that an event in a probability space holds asymptotically almost surely (or a.a.s.) if the probability that it holds tends to 1 as $n$ goes to infinity.

Let us first briefly describe some known results on the (non-lazy) cop number of $\mathcal{G}(n, p)$. Bonato, Wang, and Prałat investigated such games in $\mathcal{G}(n, p)$ random graphs and in generalizations used to model complex networks with power-law degree distributions (see [12]). From their results it follows that if $2 \log n / \sqrt{n} \leq p<1-\varepsilon$ for some $\varepsilon>0$, then a.a.s. we have that

$$
c(\mathcal{G}(n, p))=\Theta(\log n / p),
$$

so Meyniel's conjecture holds a.a.s. for such $p$. In fact, for $p=n^{-o(1)}$ we have that a.a.s. $c(\mathcal{G}(n, p))=(1+o(1)) \log _{1 /(1-p)} n$. A simple argument using dominating sets shows that Meyniel's conjecture also holds a.a.s. if $p$ tends to 1 as $n$ goes to infinity (see [22] for this and stronger results). Bollobás, Kun and Leader [5] showed that if $p(n) \geq 2.1 \log n / n$, then a.a.s. 


$$
\frac{1}{(p n)^{2}} n^{1 / 2-9 /(2 \log \log (p n))} \leq c(\mathcal{G}(n, p)) \leq 160000 \sqrt{n} \log n .
$$

From these results, if $n p \geq 2.1 \log n$ and either $n p=n^{o(1)}$ or $n p=n^{1 / 2+o(1)}$, then a.a.s. $c(\mathcal{G}(n, p))=n^{1 / 2+o(1)}$. Somewhat surprisingly, between these values it was shown by Euczak and Prałat [19] that the cop number has more complicated behaviour. It follows that a.a.s. $\log _{n} c\left(\mathcal{G}\left(n, n^{x-1}\right)\right)$ is asymptotic to the function (denoted with a solid line) shown in Figure 1.

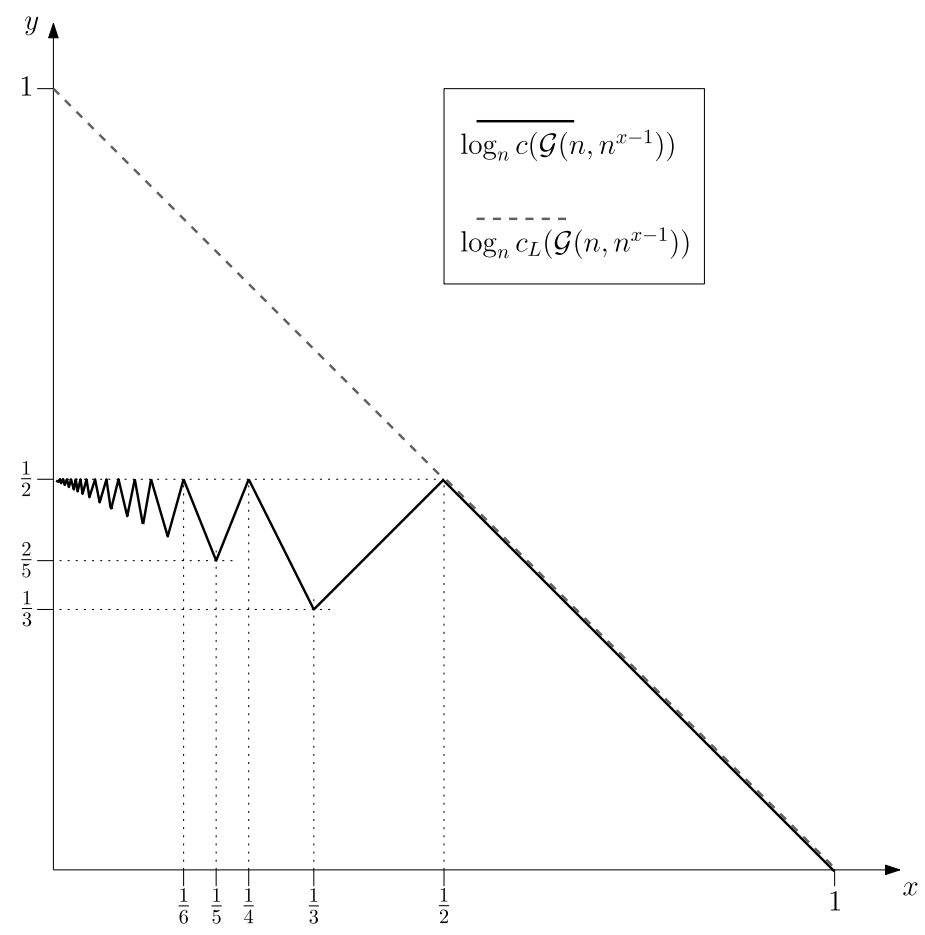

Figure 1: The "zigzag" function representing the ordinary cop number along with the function representing the lazy cop number.

The above results show that Meyniel's conjecture holds a.a.s. for random graphs except perhaps when $n p=n^{1 /(2 k)+o(1)}$ for some $k \in \mathbb{N}$, or when $n p=n^{o(1)}$. Prałat and Wormald showed recently that the conjecture holds a.a.s. in $\mathcal{G}(n, p)$ [23] as well as in random $d$-regular graphs [24].

In this paper, we investigate the lazy cop number of $\mathcal{G}(n, p)$. The main theorem of this section is the following.

Theorem 2.1. Let $0<\alpha \leq 1$, let $\varepsilon>0$, and let $d=d(n)=(n-1) p=$ $n^{\alpha+o(1)}$. 
(i) If $\alpha=1$ and $p<1-\varepsilon$, then a.a.s.

$$
c_{L}(\mathcal{G}(n, p))=(1+o(1)) \log _{1 /(1-p)} n .
$$

(Note that if $p=o(1)$, then $\log _{1 /(1-p)} n=(1+o(1)) \frac{\log n}{p}$.)

(ii) If $\frac{1}{2}<\alpha<1$, then a.a.s.

$$
c_{L}(\mathcal{G}(n, p))=\Theta\left(\frac{\log n}{p}\right) .
$$

(iii) If $\frac{1}{j+1}<\alpha<\frac{1}{j}$ for some integer $j \geq 2$, then a.a.s.

$$
\frac{1}{p}=O\left(c_{L}(\mathcal{G}(n, p))\right)=O\left(\frac{\log n}{p}\right) .
$$

(iv) If $\alpha=\frac{1}{j}$ for some integer $j \geq 2$, then a.a.s.

$$
\frac{1}{p \log ^{2} n}=O\left(c_{L}(\mathcal{G}(n, p))\right)=O\left(\frac{\log n}{p}\right) .
$$

In particular, a.a.s. $c_{L}(\mathcal{G}(n, p))=n^{1-\alpha+o(1)}$.

See Figure 1 for the corresponding function (denoted with a dotted line) representing the lazy cop number. In fact, for case (iv) we prove a slightly stronger lower bound - see Theorem 2.3 for more details.

\subsection{Upper bound}

First, let us note that $c_{L}(G) \leq \gamma(G)$ for all graphs $G$, since by initially occupying a dominating set of $G$, the cops win on their first turn. Moreover, it is well-known (and straightforward to show using, for example, the probabilistic method) that for any graph $G$

$$
\gamma(G) \leq n \frac{\log (\delta+1)}{\delta+1},
$$

where $\delta=\delta(G)$ is the minimum degree of $G$. For $G \in \mathcal{G}(n, p)$, when $p n \gg$ $\log n$ we have that a.a.s. $\delta(G)=(1+o(1)) p n$. Consequently, a.a.s.

$$
c_{L}(G) \leq(1+o(1)) \frac{\log (p n)}{p},
$$

provided that $p n \gg \log n$; this provides the upper bound for cases (ii)-(iv) in Theorem 2.1. When $p=\Omega(1)$ but $p<1-\varepsilon$ for some $\varepsilon>0$ (see case (i) 
of Theorem 2.1), one can easily show that a.a.s.

$$
\gamma(G) \leq \log _{1 /(1-p)} n+\log _{1 /(1-p)} \omega=(1+o(1)) \log _{1 /(1-p)} n
$$

where $\omega=\omega(n)=n^{o(1)}$ is any function tending to infinity sufficiently slowly as $n \rightarrow \infty$. Indeed, any set of vertices with cardinality $\log _{1 /(1-p)} n+$ $\log _{1 /(1-p)} \omega=(1+o(1)) \log _{1 /(1-p)} n$ is a.a.s. a dominating set.

\subsection{Lower bound}

For dense graphs (cases (i)-(ii) in Theorem 2.1) it is enough to use results for the classic cop number (see (1) and subsequent discussion) and the trivial observation that $c_{L}(G) \geq c(G)$.

For sparse graphs (cases (iii)-(iv) in Theorem 2.1), let us start by proving some typical properties of $\mathcal{G}(n, p)$. These observations are part of folklore, but here we provide all proofs for completeness.

Let $N_{i}[v]$ denote the set of vertices within distance $i$ of $v$. For simplicity, we use $N[v]$ to denote $N_{1}[v]$. Moreover, let $N[S]=\bigcup_{v \in S} N[v]$. Finally, let $P_{i}(v, w)$ denote the number of paths of length $i$ joining $v$ and $w$.

Lemma 2.2. Let $\varepsilon$ and $\alpha$ be constants such that $0<\varepsilon<0.1, \varepsilon<\alpha<1-\varepsilon$, and let $d=d(n)=p(n-1)=n^{\alpha+o(1)}$. Then a.a.s. for every vertex $v$ of $G=(V, E) \in \mathcal{G}(n, p)$ the following properties hold.

(i) For every $i$ such that $d^{i}=o(n)$, we have

$$
\left|N_{i}[v]\right|=(1+o(1)) d^{i}
$$

Furthermore, for $d^{i}=$ cn with $c=c(n) \leq 1$ and $c=\Omega(1)$,

$$
\left|N_{i}[v]\right|=\left(1-e^{-c}+o(1)\right) d^{i} .
$$

(ii) Let $\ell \in \mathbb{N}$ be the largest integer such that $\ell<1 / \alpha$. Then the following hold:

(a) If $w \in N_{i}[v]$ for some $i$ with $2 \leq i \leq \ell$, then $P_{i}(v, w) \leq 3 /(1-i \alpha)$.

(b) If $w \in N_{\ell+1}[v]$ and $d^{\ell+1} \geq 7 n \log n$, then $P_{\ell+1}(v, w) \leq \frac{6}{1-\ell \alpha} \frac{d^{\ell+1}}{n}$.

(c) If $w \in N_{\ell+1}[v]$ and $d^{\ell+1}<7 n \log n$, then $P_{\ell+1}(v, w) \leq \frac{42}{1-\ell \alpha} \log n$.

(d) If $w \in N_{\ell+2}[v]$ and $d^{\ell+1}<n$, then $P_{\ell+2}(v, w) \leq \frac{84}{1-\ell \alpha} \frac{d^{\ell+2} \log n}{n}$.

Proof. Let $S \subseteq V$, let $s=|S|$, and consider the random variable

$$
X=X(S)=\mid\{v \in V \backslash S: u v \in E \text { for some } u \in S\} \mid,
$$


that is, the number of vertices outside of $S$ and adjacent to at least one vertex in $S$. For (i), we bound $X$ in a stochastic sense. There are two things that need to be estimated: the expected value of $X$ and the concentration of $X$ around its expectation.

It is evident that

$$
\begin{aligned}
\mathbb{E}[X] & =\left(1-\left(1-\frac{d}{n-1}\right)^{s}\right)(n-s) \\
& =\left(1-\exp \left(-\frac{d s}{n}(1+O(d / n))\right)\right) n(1+O(s / n)) \\
& =d s(1+O(d s / n)) \\
& =d s\left(1+O\left(\log ^{-1} n\right)\right)
\end{aligned}
$$

provided $d s \leq n / \log n$. We next use a consequence of Chernoff's bound (see e.g. [16, p. 27 Cor. 2.3$])$, that

$$
\mathbb{P}(|X-\mathbb{E}[X]| \geq \varepsilon \mathbb{E}[X]) \leq 2 \exp \left(-\frac{\varepsilon^{2} \mathbb{E}[X]}{3}\right)
$$

for $0<\varepsilon<3 / 2$. This implies that the expected number of sets $S$ such that $|X(S)-d| S||>\varepsilon d|S|$ and $|S| \leq n /(d \log n)$ is, for $\varepsilon=2 / \log n$, at most

$$
\sum_{s=1}^{n /(d \log n)}\left(\begin{array}{l}
n \\
s
\end{array}\right) \cdot 2 \exp \left(-\frac{\varepsilon^{2} d s}{3+o(1)}\right) \leq \sum_{s=1}^{n /(d \log n)} 2 n^{s} \exp \left(-\frac{\varepsilon^{2} s \log ^{3} n}{3+o(1)}\right)=o(1),
$$

where the first inequality uses the fact that $d \geq \log ^{3} n$.

So a.a.s. if $|S| \leq n / d \log n$, then $X(S)=d|S|(1+O(1 / \log n))$, where the bound in $O()$ is uniform. Since $d \geq \log ^{3} n$, for such sets we have

$$
|N[S]|=|S|+X(S)=X(S)(1+O(1 / d))=d|S|(1+O(1 / \log n)) .
$$

We may assume this equation holds deterministically.

This assumption yields good bounds on the ratios of $|N[v]|$ and $|N[N[v]]|=\left|N_{2}[v]\right|$, of $\left|N_{2}[v]\right|$ and $\left|N_{3}[v]\right|$, and so on. These bounds apply to the ratio $\left|N_{r}[v]\right| /\left|N_{r-1}[v]\right|$ so long as $d^{r} \leq n / \log n$. The cumulative multiplicative error across these ratios is $\left(1+O\left(\log ^{-1} n\right)\right)^{r}$, which is $(1+o(1))$ since $r$ can be at most $1 / \alpha+o(1)=O(1)$. Thus,

$$
\left|N_{r}[v]\right|=(1+o(1)) d^{r}
$$


for all vertices $v$ and $r$ such that $d^{r} \leq n / \log n$, which establishes (i) in this case.

Suppose now that $d^{r}=c n$ with $c=c(n) \leq 1$ and $c=\Omega(1)$. Let $U=$ $N_{r-1}[v]$. Using (3), we have that $|U|=(1+o(1)) d^{r-1}$, so applying the assumption that $d^{r}=c n$, we have

$$
\begin{aligned}
\mathbb{E}[|N[U]|] & =|U|+\left(1-(1-p)^{|U|}\right)(n-|U|) \\
& =|U|+\left(1-\exp \left(-\frac{d \cdot d^{r-1}(1+o(1))}{n}(1+O(d / n))\right)\right)(n-|U|) \\
& =n\left(1-e^{-c}+o(1)\right) .
\end{aligned}
$$

Chernoff's bound (2) can be used again, in the same way as before, to show that in this case a.a.s. $|N[U]|$ is concentrated near its expected value for all $v$ and $r$. Thus, (i) holds also in this case.

The first part of (ii) can be easily verified using the first moment method. Indeed, suppose there exists $w \in N_{i}[v]$, for some $v \in V$ and $i \in \mathbb{N}$ with $2 \leq i<1 / \alpha$, such that $v$ and $w$ are joined by $k=\lceil 3 /(1-i \alpha)\rceil$ internally disjoint paths of length $i$. This structure has $2+k(i-1)$ vertices and $k i$ edges. The expected number of such subgraphs in $G$ is

$$
O\left(n^{2+k(i-1)} p^{k i}\right) \leq n^{2+k(i-1)+k i(\alpha-1)+o(1)}=n^{2-k(1-i \alpha)+o(1)} \leq n^{-1+o(1)}=o(1) .
$$

Hence, a.a.s. there is no such subgraph in $G$. Since all other possible structures joining $v$ and $w$ by $k=\lceil 3 /(1-i \alpha)\rceil$ paths of length $i$ (not necessarily internally disjoint) are even denser, the same argument applies to them as well. Finally, since $\alpha$ is constant and so is $i \leq 1 / \alpha+o(1)$, there are only finitely many structures to consider. The claim follows by the union bound.

The second part of (ii) is a consequence of (i), the first part of (ii), and Chernoff's bound. Suppose $d^{\ell+1} \geq 7 n \log n$. Let us first expose the $\ell$ th neighbourhood of $v$. By (i), we may assume that $\left|N_{\ell}[v]\right|=(1+o(1)) d^{\ell}$. For any $w \in V \backslash N_{\ell}[v]$, the expected number of edges joining $w$ to $N_{\ell}[v]$ is $p\left|N_{\ell}[v]\right|=(1+o(1)) d^{\ell+1} / n \geq(7+o(1)) \log n$. It follows from (2) that with probability $1-o\left(n^{-2}\right)$ there are at most $2 d^{\ell+1} / n$ edges joining $w$ to $N_{\ell}[v]$. By the first part of (ii), we may assume that every vertex is joined to $v$ by fewer than $3 /(1-\ell \alpha)$ paths of length $\ell$. Hence, with probability $1-o\left(n^{-2}\right)$, the desired bound on the number of $v, w$-paths of length $\ell+1$ holds for the pair $v, w$. The desired result holds by applying the union bound over all pairs under consideration. 
Suppose now that $d^{\ell+1}<7 n \log n$. This time, the expected number of edges joining $w$ and $N_{\ell}[v]$ is at most $(7+o(1)) \log n$, and we apply the more common form of Chernoff's bound: if $X$ is distributed as $\operatorname{Bin}(n, p)$, then

$$
\mathbb{P}(X \geq \mathbb{E}[X]+t) \leq \exp \left(-\frac{t^{2}}{2(\mathbb{E}[X]+t / 3)}\right)
$$

This shows that with probability $1-o\left(n^{-2}\right)$, there are at most $14 \log n$ edges joining $w$ and $N_{\ell}[v]$. The rest of the argument works as before.

Finally, suppose $d^{\ell+1}<n$. By (i), we may assume that

$$
\left(1-e^{-1}+o(1)\right) d^{\ell+1} \leq\left|N_{\ell+1}[v]\right| \leq(1+o(1)) d^{\ell+1} .
$$

The expected number of edges joining some $w \in V \backslash N_{\ell+1}[v]$ to $N_{\ell+1}[v]$ is $p\left|N_{\ell+1}[v]\right|=\Theta\left(d^{\ell+2} / n\right) \geq(7+o(1)) \log n$. It follows from (2) that with probability $1-o\left(n^{-2}\right)$ there are at most $2 d^{\ell+2} / n$ edges joining $w$ to $N_{\ell+1}[v]$, and the desired bound holds.

Now, we are ready to prove our lower bound on $c_{L}(G)$ for $G \in \mathcal{G}(n, p)$. The proof is an adaptation of the proof used for the classic cop number in [19]. Let us point out that in this paper we also deal with the case $\alpha=$ $1 /(j+1)$, which was omitted in [19].

Theorem 2.3. Let $\frac{1}{j+1}<\alpha<\frac{1}{j}$ for some $j \in \mathbb{N}, c=c(j, \alpha)=\frac{6}{1-j \alpha}$, and $d=d(n)=p(n-1)=n^{\alpha+o(1)}$. Then a.a.s. for $G=(V, E) \in \mathcal{G}(n, p)$ we have that

$$
c_{L}(G) \geq \frac{1-j \alpha}{12(2 c)^{j-1} j^{j}} \cdot \frac{1}{p}=\Omega\left(\frac{1}{p}\right) .
$$

Let $\alpha=\frac{1}{j+1}$ for some $j \in \mathbb{N}$. Then a.a.s. for $G=(V, E) \in \mathcal{G}(n, p)$ we have that

$$
c_{L}(G) \geq \begin{cases}\frac{1-j \alpha}{12(2 c)^{j-1} j^{j}} \cdot \frac{1}{p}=\Omega\left(\frac{1}{p}\right), & \text { if } d^{j+1} \geq 7 n \log n \\ \frac{c(1-j \alpha)}{42(2 c j)^{j}} \cdot \frac{d^{j}}{\log n}=\Omega\left(\frac{1}{p^{2} \log n}\right), & \text { if } \frac{n}{\log n} \leq d^{j+1}<7 n \log n \\ \frac{c^{2}(1-j \alpha)^{2}}{3528(2 c(j+1))^{j+1}} \cdot \frac{n}{d \log ^{2} n}=\Omega\left(\frac{1}{p \log ^{2} n}\right), & \text { if } d^{j+1}<n / \log n .\end{cases}
$$


Proof. In all cases, we provide a winning strategy for the robber on $G$. Since our aim is to prove that the bounds hold a.a.s., we may assume without loss of generality that $G$ satisfies the properties stated in Lemma 2.2.

Suppose first that $1 /(j+1)<\alpha<1 / j$ and that the robber is chased by $K=\frac{1-j \alpha}{12(2 c)^{j-1} j^{j}} \cdot \frac{1}{p}$ cops. Let $\mathrm{C}_{i}(v)$ denote the number of cops in $N_{i}[v]$; in particular, $\mathrm{C}_{0}(v)=0$ if and only if $v$ is not occupied by a cop. Right before the cops make their move, we say that the vertex $v$ occupied by the robber is safe if we have $\mathrm{C}_{0}(v)=0, \mathrm{C}_{1}(v)=0$, and

$$
\mathrm{C}_{i}(v) \leq\left(\frac{d}{2 c j}\right)^{i-1}
$$

for $i=2,3, \ldots, j$.

Since a.a.s. $G$ is connected, without loss of generality we may assume that at the beginning of the game all cops begin at the same vertex, $z$. Subsequently, the robber may choose a vertex $v$ at distance $j+1$ from $z$ (see Lemma 2.2(i) with $i=j$ ); clearly $v$ is safe. Hence, in order to prove the theorem, it is enough to show that if the robber's current vertex is safe, then no matter how the cops move in the next round, the robber can always move to a safe vertex.

For $r \geq 0$, we say that a neighbour $y$ of $v$ is $r$-dangerous if

(i) $\mathrm{C}_{r}(y)>0$ (for $\left.r=0,1\right)$, or

(ii) $\mathrm{C}_{r}(y)>\left(\frac{d}{2 c j}\right)^{r-1}$ (for $r=2,3, \ldots, j$ ).

Clearly, no neighbour of $v$ is 0 -dangerous (since $v$ is safe, $\mathrm{C}_{1}(v)=0$ ). We now check that for every $r \in\{1,2, \ldots, j\}$, the number of $r$-dangerous neighbours of $v$, which we denote by $\operatorname{dang}(r)$, is smaller than $d /(2 j)$. Every 1-dangerous vertex has a cop as a neighbour. On the other hand, every cop is adjacent to at most $c$ neighbours of $v$, since otherwise we would have more than $c$ paths between this cop and $v$, contradicting Lemma 2.2(ii). Moreover, by the assumption that $v$ is safe, we have $\mathrm{C}_{2}(v) \leq \frac{d}{2 c j}$. Combining all of these yields

$$
\operatorname{dang}(1) \leq c \cdot \mathrm{C}_{2}(v) \leq c\left(\frac{d}{2 c j}\right)=\frac{d}{2 j} .
$$

For $r \in\{2,3, \ldots, j-1\}$, we consider pairs $(y, w)$ where $y$ is an $r$-dangerous neighbour of $v$ and $w$ is a cop at distance $r$ from $y$. If $3 \leq r+1 \leq j$, then Lemma 2.2(ii) implies that there are at most $c$ paths between $v$ and $w$. It follows that fewer than $c$ neighbours of $v$ are a distance $r$ from $w$. Estimating 
the number of pairs $(y, w)$ in two ways, we have

$$
\left(\frac{d}{2 c j}\right)^{r-1} \operatorname{dang}(r) \leq c \cdot \mathrm{C}_{r+1}(v) \leq c\left(\frac{d}{2 c j}\right)^{r},
$$

and consequently $\operatorname{dang}(r) \leq \frac{d}{2 j}$.

Checking the desired bound for $\operatorname{dang}(j)$ is slightly more complicated. This time, a cop at distance $j+1$ from $v$ can contribute to the "dangerousness" of more than $c$ neighbours of $v$. However, the number of paths of length $j+1$ joining $v$ and $w$ is bounded from above by $\frac{6}{1-j \alpha} \cdot \frac{d^{j+1}}{n}$ (see Lemma 2.2(ii) and note that $d^{j+1}=n^{\alpha(j+1)+o(1)} \geq 7 n \log n$, since $\left.\alpha(j+1)>1\right)$. Although we cannot control the number of cops in $N_{j+1}[v]$, clearly $\mathrm{C}_{j+1}(v)$ is bounded from above by $K=\frac{1-j \alpha}{12(2 c)^{j-1} j^{j}} \cdot \frac{n}{d}$, the total number of cops. Hence,

$$
\left(\frac{d}{2 c j}\right)^{j-1} \operatorname{dang}(j) \leq \frac{6 d^{j+1}}{(1-j \alpha) n} \cdot K=c\left(\frac{d}{2 c j}\right)^{j}
$$

and, as desired, $\operatorname{dang}(j) \leq \frac{d}{2 j}$. It follows that at most $d / 2$ neighbours of $v$ are $r$-dangerous for some $r=0,1, \ldots, j$.

Now, it is time for the cops to make their move. Fortunately, only one cop may move, and this single cop can cause at most $c$ neighbours of $v$ to become $r$-dangerous for some $r<j$. Finally, we may use Lemma 2.2(i) to infer that there is a neighbour $y$ of $v$ that is not $r$-dangerous for any $r=0,1, \ldots, j$. The vertex $y$ is safe; we move the robber there. This completes the proof of (5).

Suppose now that $\alpha=1 /(j+1)$ for some $j \in \mathbb{N}$. The argument for this case is quite similar, so we only mention the differences. We consider three cases. First, suppose $d^{j+1} \geq 7 n \log n$. Since the number of paths of length $j+1$ from $v$ to a vertex $w$ is bounded from above by the same value, namely $\frac{6}{1-j \alpha} \cdot \frac{d^{j+1}}{n}$ (see Lemma 2.2(ii)), the calculations (and hence also the bound) are exactly the same.

Second, suppose $n / \log n \leq d^{j+1}<7 n \log n$. In this case, the number of paths of length $j+1$ from $v$ to $w$ is bounded above by $\frac{42}{1-j \alpha} \log n$ (as before, see Lemma 2.2(ii)). Hence, we must replace (6) by

$$
\left(\frac{d}{2 c j}\right)^{j-1} \operatorname{dang}(j) \leq \frac{42}{1-j \alpha} \log n \cdot K=c\left(\frac{d}{2 c j}\right)^{j},
$$

provided that $K$ is adjusted to be $\frac{c(1-j \alpha)}{42(2 c j)^{j}} \cdot \frac{d^{j}}{\log n}$. 
Third, suppose $d^{j+1}<n / \log n$. This time, the adjustments are slightly more complicated, since we must control the number of cops within distance $j+1$ of the robber. In particular, we need to find a neighbour $y$ of $v$ that is not $r$-dangerous for any $r=0,1, \ldots, j+1$. We adjust the definition of being "safe" as follows: $\mathrm{C}_{0}(v)=0, \mathrm{C}_{1}(v)=0, \mathrm{C}_{i}(v) \leq\left(\frac{d}{2 c(j+1)}\right)^{i-1}$ for every $i=2,3, \ldots, j$, and $\mathrm{C}_{j+1}(v) \leq\left(\frac{d}{2 c(j+1)}\right)^{j} \frac{c(1-j \alpha)}{42 \log n}$. This assures that $\operatorname{dang}(j)$ is bounded as needed, that is,

$$
\left(\frac{d}{2 c(j+1)}\right)^{j-1} \operatorname{dang}(j) \leq \frac{42}{1-j \alpha} \log n \cdot \mathrm{C}_{j+1}(v)=c\left(\frac{d}{2 c(j+1)}\right)^{j} .
$$

Finally, the number of $(j+1)$-dangerous neighbours of $v$ can be given by

$$
\left(\frac{d}{2 c(j+1)}\right)^{j} \frac{c(1-j \alpha)}{42 \log n} \cdot \operatorname{dang}(j+1) \leq \frac{84}{1-j \alpha} \cdot \frac{d^{j+2} \log n}{n} \cdot K,
$$

so $\operatorname{dang}(j+1) \leq \frac{d}{2(j+1)}$, provided that $K$ is adjusted to be $\frac{c^{2}(1-j \alpha)^{2}}{3528(2 c(j+1))^{j+1}} \cdot \frac{n}{d \log ^{2} n}$.

\section{Graphs on surfaces}

The genus of a graph $G$ is the minimum genus of an orientable surface on which $G$ can be embedded without edge crossings. Graphs with genus 0 are the planar graphs, and it was shown in [1] that planar graphs have cop number at most 3. If $G$ has genus $g$, then it was proved in [26] that $c(G) \leq\left\lfloor\frac{3 g}{2}\right\rfloor+3$. In the same paper, it was conjectured that $c(G) \leq g+3$.

We conclude the paper with a straightforward asymptotic upper bound on $c_{L}$ for graphs with genus $g$. We use a well-known separator result due to Gilbert, Hutchinson, and Tarjan [15].

Theorem 3.1 ([15]). Every $n$-vertex graph of genus $g$ contains set of at most $6 \sqrt{g n}+2 \sqrt{2 n}+1$ vertices whose removal leaves a graph in which no component has more than $2 n / 3$ vertices.

We obtain our bound on $c_{L}$ as a direct consequence of Theorem 3.1.

Theorem 3.2. For every $n$-vertex graph $G$ of genus $g$ we have $c_{L}(G) \leq$ $60 \sqrt{g n}+20 \sqrt{2 n}=O(\sqrt{g n})$.

Proof. Let $K=60 \sqrt{g n}+20 \sqrt{2 n}=O(\sqrt{g n})$; we use induction on $n$ to prove that $c_{L}(G) \leq K$. When $n=1$, we have that $c_{L}(G)=1$, so the bound holds. 
Assume $n \geq 2$, and suppose that the bound holds for all graphs on fewer than $n$ vertices.

By Theorem 3.1, $G$ contains some separating set $S$ of cardinality at most $6 \sqrt{g n}+2 \sqrt{2 n}+1$, such that each component of $G-S$ has at most $2 n / 3$ vertices. The cops play as follows. First, one cop occupies each vertex of $S$. If the robber has not yet been captured, then he must inhabit some component $X$ of $G-S$. The cops currently occupying vertices of $S$ remain in place for the duration of the game; consequently, the robber cannot leave $X$ without being captured. The remaining $K-|S|$ cops now move to $X$ and, subsequently, attempt to capture the robber while remaining within $X$. By choice of $X$ and the induction hypothesis, these cops may capture the robber so long as

$$
K-|S| \geq 60 \sqrt{g \frac{2 n}{3}}+20 \sqrt{2 \frac{2 n}{3}} .
$$

Since $|S| \leq 6 \sqrt{g n}+2 \sqrt{2 n}+1$, it suffices to show that

$$
K \geq 60 \sqrt{g \frac{2 n}{3}}+20 \sqrt{2 \frac{2 n}{3}}+6 \sqrt{g n}+2 \sqrt{2 n}+1 .
$$

However,

$$
\begin{aligned}
60 & \sqrt{g \frac{2 n}{3}}+20 \sqrt{2 \frac{2 n}{3}}+6 \sqrt{g n}+2 \sqrt{2 n}+1 \\
& =\left(60 \sqrt{\frac{2}{3}}+6\right) \sqrt{g n}+\left(20 \sqrt{\frac{2}{3}}+2\right) \sqrt{2 n}+1 \\
& <55 \sqrt{g n}+19 \sqrt{2 n}+1<K,
\end{aligned}
$$

which completes the proof.

It is not known whether the bounds in Theorem 3.2 are asymptotically tight, even in the case of planar graphs. In fact, we are not presently aware of any families of planar graphs on which the lazy cop number grows as an unbounded function.

\section{References}

[1] M. Aigner, M. Fromme, A game of Cops and Robbers, Discrete Applied Mathematics 8 (1984) 1-12. MR0739593 
[2] N. Alon, A. Mehrabian, Chasing a fast robber on planar graphs and random graphs, Journal of Graph Theory 78 (2015) 81-96. MR3293077

[3] W. Baird, A. Bonato, Meyniel's conjecture on the cop number: a survey, Journal of Combinatorics 3 (2012) 225-238. MR2980752

[4] D. Bal, A. Bonato, W. Kinnersley, P. Prałat, Lazy Cops and Robbers on hypercubes, Combinatorics, Probability and Computing 24 (2015), 829-837. MR3406447

[5] B. Bollobás, G. Kun, I. Leader, Cops and Robbers in a random graph, Journal of Combinatorial Theory Series B 103 (2013) 226-236. MR3018067

[6] A. Bonato, What is ... cop number? Notices of the American Mathematical Society 59 (2012) 1100-1101. MR2985811

[7] A. Bonato, Catch me if you can: Cops and Robbers on graphs, In: Proceedings of the 6th International Conference on Mathematical and Computational Models (ICMCM'11), 2011.

[8] A. Bonato, E. Chiniforooshan, Pursuit and evasion from a distance: algorithms and bounds, In: Proceedings of ANALCO'09, 2009. MR2648337

[9] A. Bonato, E. Chiniforooshan, P. Prałat, Cops and Robbers from a distance, Theoretical Computer Science 411 (2010) 3834-3844. $\operatorname{MR} 2779754$

[10] A. Bonato, S. Finbow, P. Gordinowicz, A. Haidar, W.B. Kinnersley, D. Mitsche, P. Prałat, L. Stacho. The robber strikes back, In: Proceedings of the International Conference on Computational Intelligence, Cyber Security and Computational Models (ICC3), 2013.

[11] A. Bonato, R.J. Nowakowski, The Game of Cops and Robbers on Graphs, American Mathematical Society, Providence, Rhode Island, 2011. MR2830217

[12] A. Bonato, P. Prałat, C. Wang, Network security in models of complex networks, Internet Mathematics 4 (2009) 419-436.

[13] A. Dudek, P. Gordinowicz, P. Prałat, Cops and Robbers playing on edges, Journal of Combinatorics 5 (2014), 131-153. MR3173800

[14] A. Frieze, M. Krivelevich, P. Loh, Variations on Cops and Robbers, Journal of Graph Theory 69, 383-402. MR2979296 
[15] J.R. Gilbert, J.P. Hutchinson, R.E. Tarjan, A separator theorem for graphs of bounded genus, J. Algorithms 5 (1984) 391-407. MR0756165

[16] S. Janson, T. Łuczak, A. Ruciński, Random Graphs, Wiley, New York, 2000. MR1782847

[17] A. Kehagias, D. Mitsche, P. Prałat, Cops and invisible robbers: the cost of drunkenness, Theoretical Computer Science 481 (2013), 100120. MR3037800

[18] A. Kehagias, P. Prałat, Some remarks on cops and drunk robbers, Theoretical Computer Science 463 (2012), 133-147. MR2992504

[19] T. Łuczak, P. Prałat, Chasing robbers on random graphs: zigzag theorem, Random Structures and Algorithms 37 (2010) 516-524. MR2760362

[20] R.J. Nowakowski, P. Winkler, Vertex-to-vertex pursuit in a graph, Discrete Mathematics 43 (1983) 235-239. MR0685631

[21] D. Offner, K. Okajian, Variations of Cops and Robber on the hypercube, Australasian Journal of Combinatorics 59 (2014), 229-250. MR3245401

[22] P. Prałat, When does a random graph have constant cop number? Australasian Journal of Combinatorics 46 (2010) 285-296. MR2598712

[23] P. Prałat, N.C. Wormald, Meyniel's conjecture holds for random graphs, Random Structures and Algorithms 48 (2016), 396-421. MR3449604

[24] P. Prałat, N.C. Wormald, Meyniel's conjecture holds for random $d$ regular graphs, preprint 2014.

[25] A. Quilliot, Jeux et pointes fixes sur les graphes, Thèse de 3ème cycle, Université de Paris VI, 1978, 131-145.

[26] B.S.W. Schroeder, The copnumber of a graph is bounded by $\left\lfloor\frac{3}{2}\right.$ genus $\left.(G)\right\rfloor+3$, Categorical perspectives (Kent, OH, 1998), Trends Math., Birkhäuser Boston, Boston, MA, 2001, 243-263. MR1827672

[27] D.B. West, Introduction to Graph Theory, 2nd edition, Prentice Hall, 2001 MR1367739

DEEPAK BAL

Department of Mathematical Sciences

Montclair State University

MONTCLAIR, NJ 07042

USA

E-mail address: deepak.bal@montclair.edu 


\author{
AnTHONY Bonato \\ Department of Mathematics \\ RYERSON UNIVERSITY \\ Toronto, ON M5B 2K3 \\ CANADA \\ E-mail address: abonato@ryerson.ca \\ William B. Kinnersley \\ Department of Mathematics \\ UNIVERSITY OF RHODE IsLAND \\ Kingston, RI 02881 \\ USA \\ E-mail address: billk@uri.edu \\ Pawee Praeat \\ Department of Mathematics \\ RYERSON UNIVERSITY \\ Toronto, ON M5B 2K3 \\ CANADA \\ E-mail address: pralat@ryerson.ca \\ ReCEIVED 30 May 2014
}

